Supporting Information (SI)

\title{
Acetylene Separation by a Ca-MOF Containing Accessible Sites of Open Metal Centers and Organic Groups
}

Gang-Ding Wang ${ }^{\dagger}$, Yong-Zhi Li ${ }^{\dagger}$, Wan-Fang Zhang ${ }^{\dagger}$, Lei Hou, ${ }^{*}{ }^{\dagger}$ Yao-Yu Wang ${ }^{\dagger}$ and Zhonghua Zhu ${ }^{\ddagger}$

†Key Laboratory of Synthetic and Natural Functional Molecule Chemistry of the Ministry of Education,

Shaanxi Key Laboratory of Physico-Inorganic Chemistry, College of Chemistry \& Materials Science,

Northwest University, Xi'an 710069, P. R. China.

tSchool of Chemical Engineering, The University of Queensland, Brisbane 4072, Australia.

*To whom correspondence should be addressed. E-mail: 1hou2009@nwu.edu.cn (Lei Hou). 


\section{Materials and General Methods}

All solvents for synthesis were purchased commercially. Elemental analyses of $\mathrm{C}, \mathrm{H}$, and $\mathrm{N}$ were determined with a Perkin-Elmer 2400C elemental analyzer. Infrared spectra were obtained in $\mathrm{KBr}$ discs on a Nicolet Avatar 360 FTIR spectrometer in the $400-4000 \mathrm{~cm}^{-1}$ region. Powder X-ray diffraction (PXRD) data were recorded on a Bruker D8 ADVANCE X-ray powder diffractometer $(\mathrm{Cu} \mathrm{K} \alpha, \lambda=1.5418 \AA)$. Thermalgravimetric analyses (TGA) were carried out in a nitrogen stream using a Netzsch TG209F3 equipment at a heating rate of $10{ }^{\circ} \mathrm{C} \mathrm{min}^{-1}$. Adsorption measurements were performed with an automatic volumetric sorption apparatus (Micrometrics ASAP 2020M). Breakthrough experiments were performed on a Quantachrome dynaSorb BT equipments.

\section{X-Ray Crystallography}

A Bruker Smart Apex II CCD detector was used to collect the single crystal data at 150(2) $\mathrm{K}$ using Mo K $\alpha$ radiation $(\lambda=0.71073 \AA)$. The structure was solved by direct methods and refined by full-matrix least-squares refinement based on $\mathrm{F}^{2}$ with the SHELXTL program. The non-hydrogen atoms were refined anisotropically with the hydrogen atoms added at their geometrically ideal positions and refined isotropically. As the disordered solvent molecules in the structure cannot be located, the SQUEEZE routine of Platon program was applied in refining. Relevant crystallographic results are listed in Table S1. Selected bond lengths and angles are provided in Table S2.

\section{Gas Sorption Experiments}

Before gas sorption experiments, the as-synthesized sample was immersed in $\mathrm{CH}_{2} \mathrm{Cl}_{2}$ for 3 days, during which the solvent was decanted and freshly replenished three times one day. All sample was activated under vacuum at $523 \mathrm{~K}$ for 4 hours. The activated sample 1a indicated a color change from white to yellow (Figure S4). Gas sorption measurements were then conducted using a Micrometrics ASAP 2020M gas adsorption analyzer.

\section{Breakthrough Experiments}

The breakthrough experiment was performed on the Quantachrome dynaSorb BT 
equipments at $298 \mathrm{~K}$ and 1 bar with an equal volume of mixed gas (gas $\mathrm{A}$ : gas $\mathrm{B}: \mathrm{Ar}=5 \%$ : 5\%: $90 \%$ or gas A: gas B: gas C: $\mathrm{Ar}=5 \%: 5 \%: 5 \%: 85 \%$, Ar as the carrier gas, flow rate $=7$ $\left.\mathrm{mL} \min ^{-1}\right)$. The activated $1 \mathrm{a}(0.85 \mathrm{~g})$ was filled into a packed column of $\phi 4.2 \times 80 \mathrm{~mm}$, and then the packed column was washed with Ar at a rate of $7 \mathrm{~mL} \mathrm{~min}^{-1}$ at $343 \mathrm{~K}$ for 60 minutes to further activate the samples. Between two breakthrough experiments, the adsorbent was regenerated by Ar flow of $7 \mathrm{~mL} \mathrm{~min}^{-1}$ for $35 \mathrm{~min}$ at $353 \mathrm{~K}$ to guarantee a complete removal of the adsorbed gases.

\section{GCMC Simulation}

Grand canonical Monte Carlo (GCMC) simulations were performed for the gas adsorption in the framework by the Sorption module of Material Studio (Accelrys. Materials Studio Getting Started). The framework was considered to be rigid, and the optimized gas molecules were used. The atom charges and bond lengths for the gas molecules are as follows:

$$
\begin{aligned}
& \mathrm{CO}_{2}, \mathrm{C}=0.576000 \mathrm{e}, \mathrm{O}=-0.288000 \mathrm{e}, \mathrm{C}-\mathrm{O}=1.168 \AA . \\
& \mathrm{C}_{2} \mathrm{H}_{2}, \mathrm{C}=-0.238847 \mathrm{e}, \mathrm{H}=0.238847 \mathrm{e}, \mathrm{C}-\mathrm{C}=1.198 \AA, \mathrm{C}-\mathrm{H}=1.065 \AA . \\
& \mathrm{C}_{2} \mathrm{H}_{4}, \mathrm{C}=-0.295071 \mathrm{e}, \mathrm{H}=0.147536 \mathrm{e}, \mathrm{C}-\mathrm{C}=1.334 \AA, \mathrm{C}-\mathrm{H}=1.090 \AA . \\
& \mathrm{C}_{2} \mathrm{H}_{6}, \mathrm{C}=-0.458757 \mathrm{e}, \mathrm{H}=0.152919 \mathrm{e}, \mathrm{C}-\mathrm{C}=1.528 \AA, \mathrm{C}-\mathrm{H}=1.099 \AA .
\end{aligned}
$$

The partial charges for atoms of the framework were derived from QEq method and QEq neutral 1.0 parameter. One unit cell was used during the simulations. The interaction energies between the gas molecules and framework were computed through the Coulomb and Lennard-Jones 6-12 (LJ) potentials. All parameters for the atoms were modeled with the universal force field (UFF) embedded in the MS modeling package. A cutoff distance of 12.5 $\AA$ was used for LJ interactions, and the Coulombic interactions were calculated by using Ewald summation. For each run, the $3 \times 10^{6}$ maximum loading steps, $3 \times 10^{6}$ production steps were employed.

The binding energy was calculated by DFT method using the Dmol3 software. The exchange-correlation functional used in calculations was in the framework of the generalized gradient approximation (GGA) proposed by Perdew, Burke and Ernzerhof (PBE). DNP basis set was used to describe the atomic orbital. The SCF convergence was set to $10^{-5}$. The binding energy is evaluated by the following equation: $E_{\text {bind }}=E_{\text {framework }+ \text { gas }}-E_{\text {framework }}-E_{\text {gas }}$, in which 
$E_{\text {framework }+ \text { gas }}$ is the total energy of the framework and the adsorbed gas molecule, $E_{\text {framework }}$ and $E_{\text {gas }}$ are the energies of the framework and gas molecule.

\section{Fitting Adsorption Heat of Pure Component Isotherms}

$$
\ln P=\ln N+1 / T \sum_{i=0}^{m} a_{i} N^{i}+\sum_{i=0}^{n} b_{i} N^{i} \quad Q_{\mathrm{st}}=-R \sum_{i=0}^{m} a_{i} N^{i}
$$

The above virial expression was used to fit the combined isotherm data for 1a at 273.15 and $298 \mathrm{~K}$, where $\mathrm{P}$ is the pressure, $\mathrm{N}$ is the adsorbed amount, $\mathrm{T}$ is the temperature, ai and bi are virial coefficients, and $\mathrm{m}$ and $\mathrm{N}$ are the number of coefficients used to describe the isotherms. $Q_{\mathrm{st}}$ is the coverage-dependent enthalpy of adsorption and $\mathrm{R}$ is the universal gas constant.

\section{Gas Selectivity Prediction via IAST}

The experimental isotherm data for pure gas were fitted using a dual Langmuir-Freundlich (L-F) model:

$$
q=\frac{a_{1} * b_{1} * P^{c_{1}}}{1+b_{1} * P^{c_{1}}}+\frac{a_{2} * b_{2} * P^{c_{2}}}{1+b_{2} * P^{c_{2}}}
$$

Where $\mathrm{q}$ and $\mathrm{p}$ are adsorbed amounts and the pressure of component $\mathrm{i}$, respectively.

The adsorption selectivity for binary mixture, defined by

$$
S_{\mathrm{i} / \mathrm{j}}=\frac{x_{\mathrm{i}}^{*} y_{\mathrm{j}}}{x_{\mathrm{j}}^{*} y_{\mathrm{i}}}
$$

was respectively calculated using the Ideal Adsorption Solution Theory (IAST). Where $\mathrm{x}_{\mathrm{i}}$ is the mole fraction of component $\mathrm{i}$ in the adsorbed phase and $\mathrm{y}_{\mathrm{i}}$ is the mole fraction of component $i$ in the bulk. 
a)

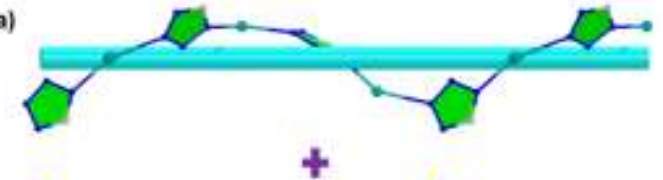

b)

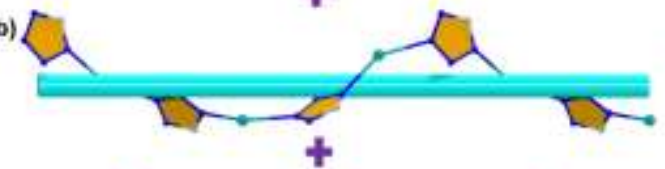

c)

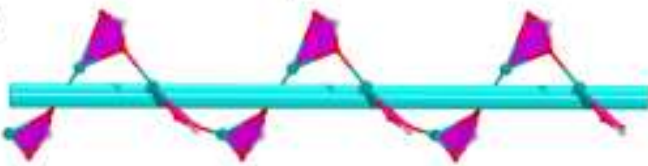

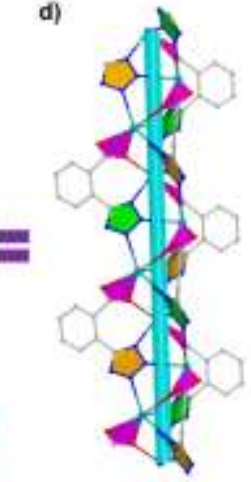

Figure S1. Metal-organic chain SBU with a threefold helix in $\mathbf{1 .}$

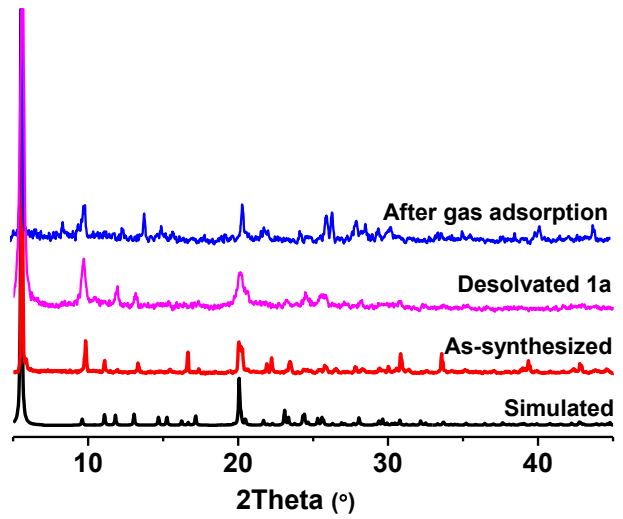

Figure S2. PXRD patterns of complex 1.

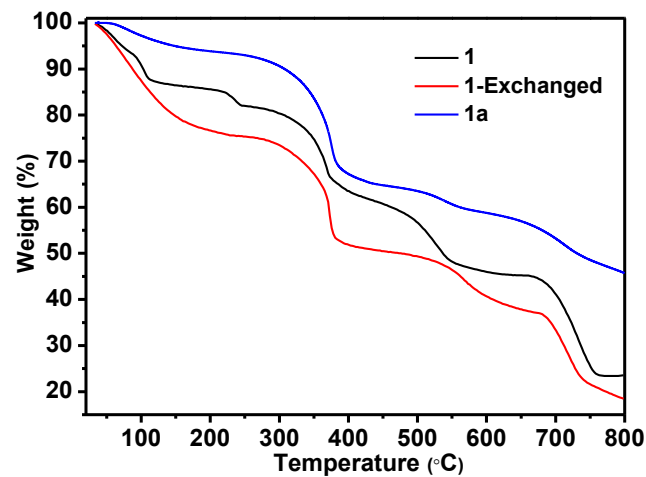

Figure S3. TGA curves of as-synthesized, $\mathrm{CH}_{2} \mathrm{Cl}_{2}$-exchanged and activated samples of complex 1 . 


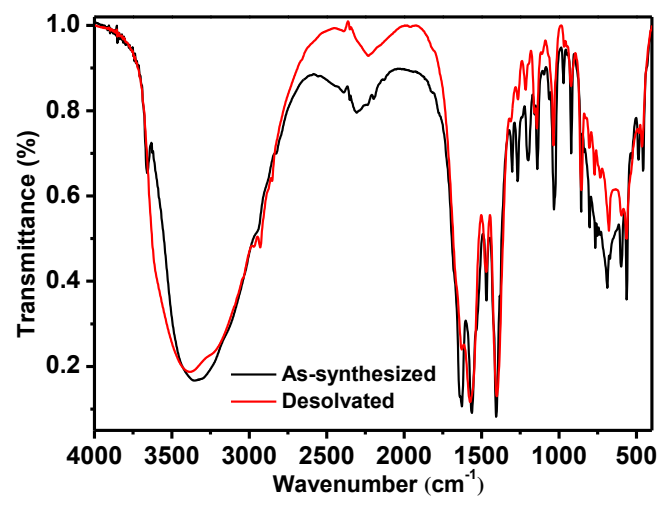

Figure S4. FTIR spectra of the as-synthesized and activated samples of $\mathbf{1}$.

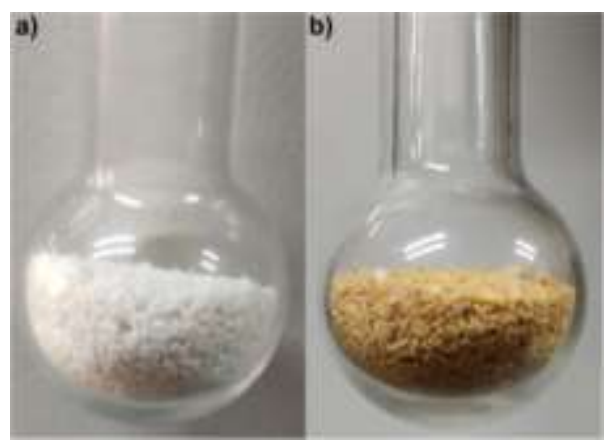

Figure S5. Color changes of the as-synthesized (a) and activated (b) samples of $\mathbf{1}$.

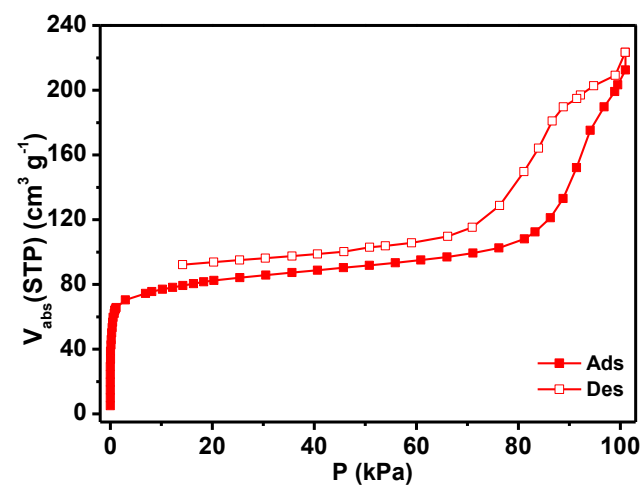

Figure S6. $\mathrm{N}_{2}$ sorption isotherm of $1 \mathrm{a}$ at $77 \mathrm{~K}$.

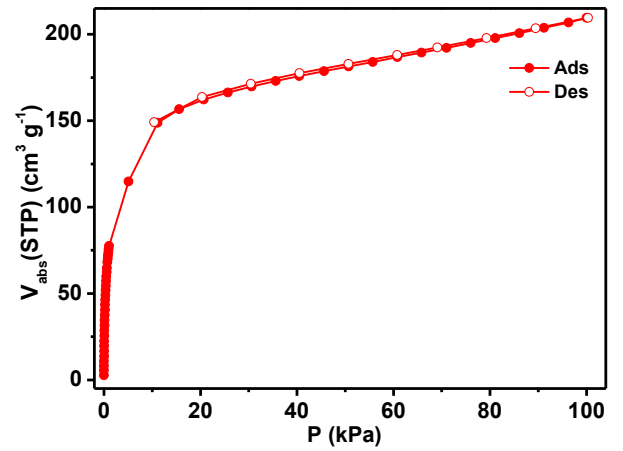

Figure $\mathbf{S 7} . \mathrm{CO}_{2}$ sorption isotherm of $1 \mathrm{a}$ at $195 \mathrm{~K}$. 

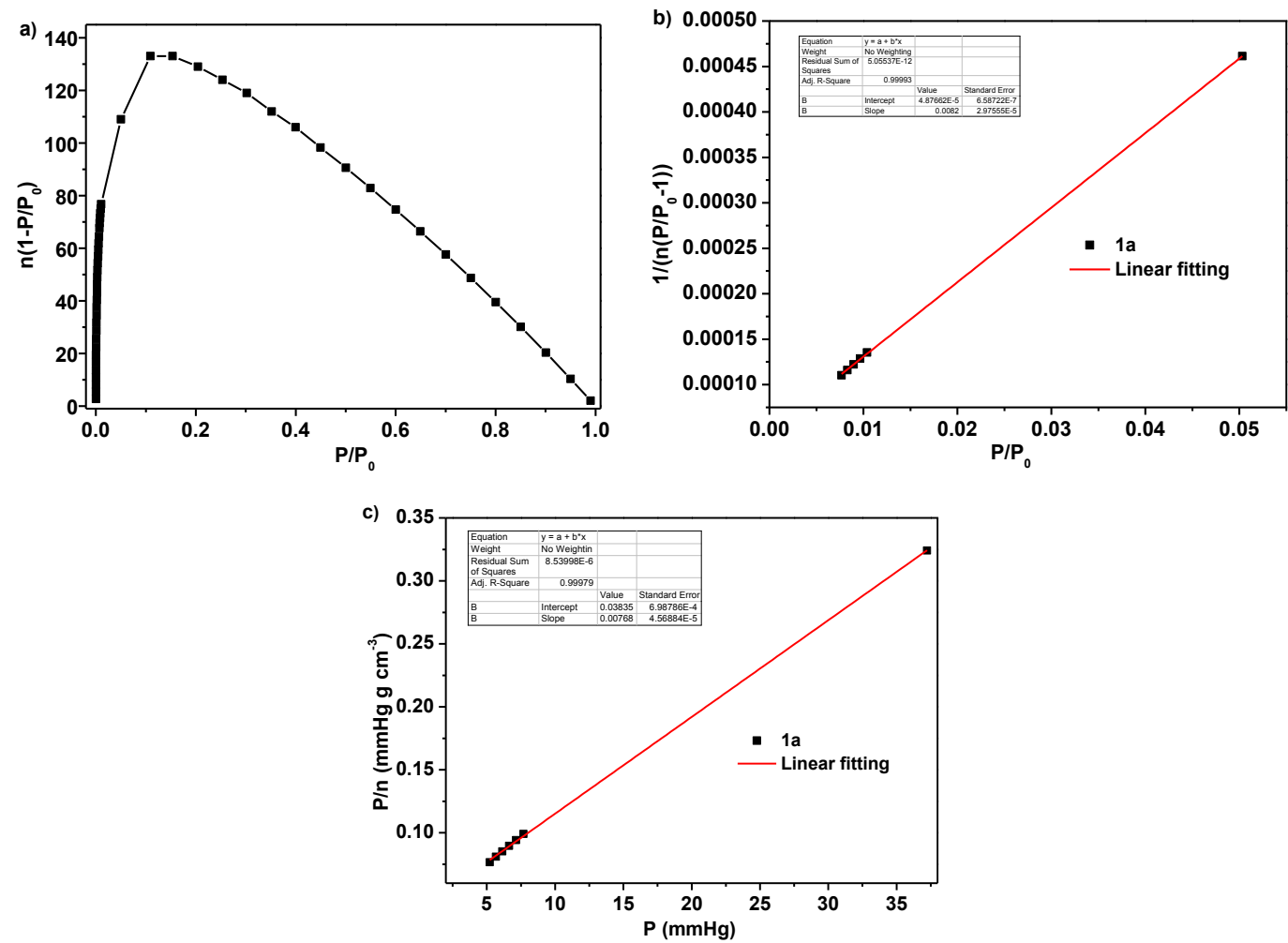

Figure S8. The consistency plot (a), BET surface area plot (b), and Langmuir surface area plot (c), from the $\mathrm{CO}_{2}$ adsorption isotherm at $195 \mathrm{~K}$ for $1 \mathbf{a}$.

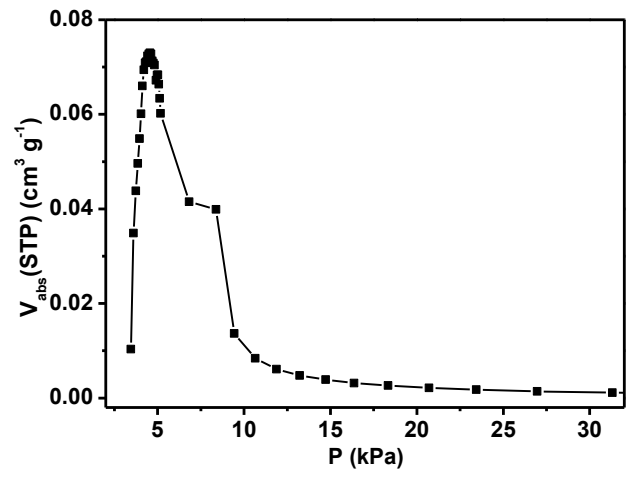

Figure S9. Differential pore volume in 1a as a function of pore width calculated from the $\mathrm{CO}_{2}$ adsorption isotherm at $195 \mathrm{~K}$ using the Horvath-Kawazoe model. 


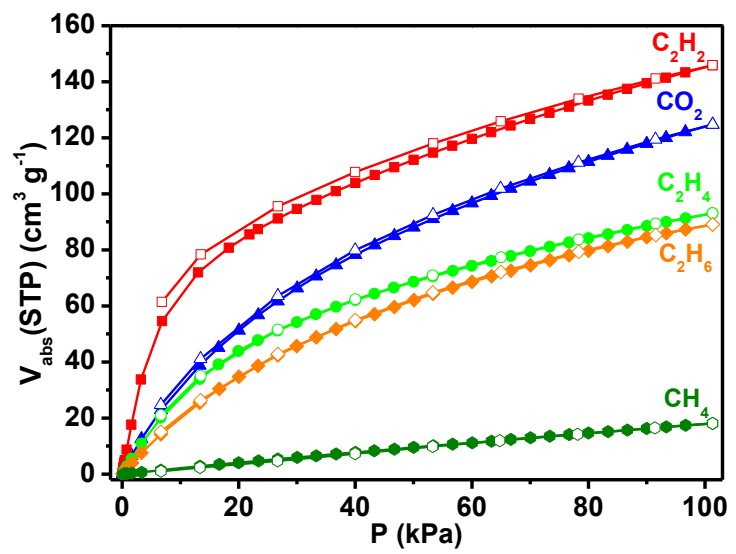

Figure S10. gas adsorption isotherms for $\mathrm{CO}_{2}, \mathrm{C}_{2} \mathrm{H}_{6}, \mathrm{C}_{2} \mathrm{H}_{4}, \mathrm{C}_{2} \mathrm{H}_{2}$ and $\mathrm{CH}_{4}$ at $273 \mathrm{~K}$.
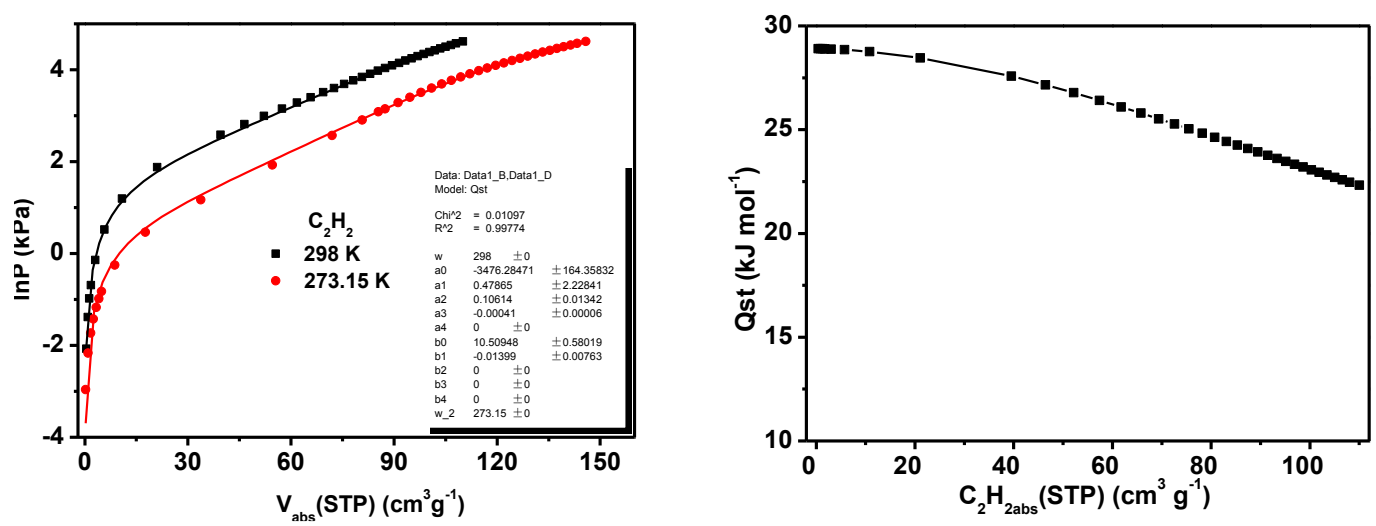

Figure S11. Fitted $\mathrm{C}_{2} \mathrm{H}_{2}$ isotherms of 1a measured at 273.15 and $298 \mathrm{~K}$, and the corresponding isosteric heats of adsorption $\left(Q_{\mathrm{st}}\right)$.
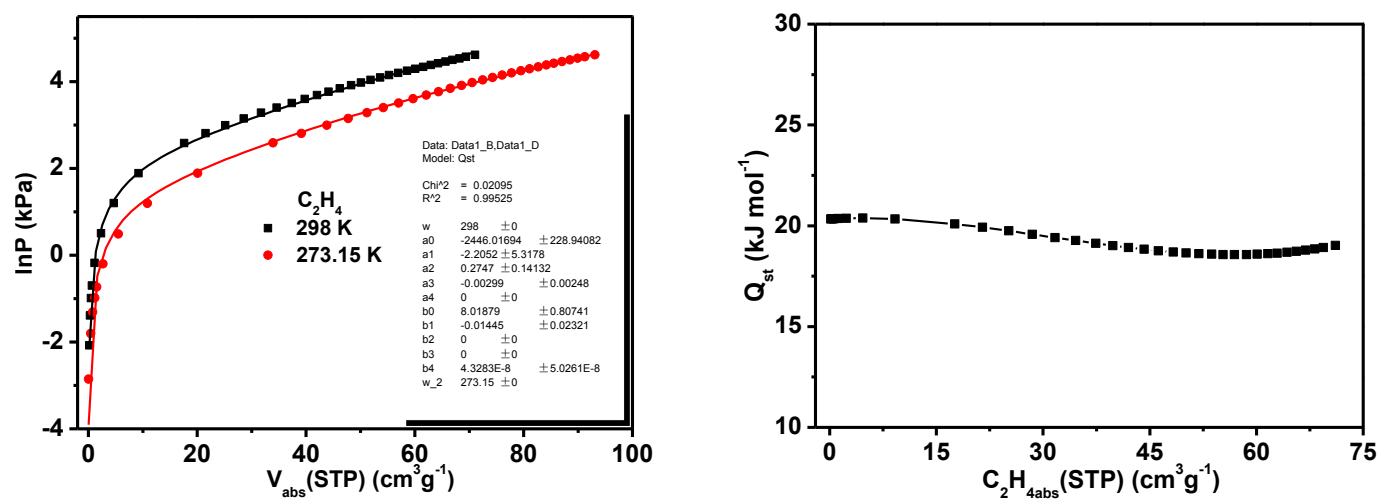

Figure S12. Fitted $\mathrm{C}_{2} \mathrm{H}_{4}$ isotherms of $1 \mathrm{a}$ measured at 273.15 and $298 \mathrm{~K}$, and the corresponding isosteric heats of adsorption $\left(Q_{\text {st }}\right)$. 

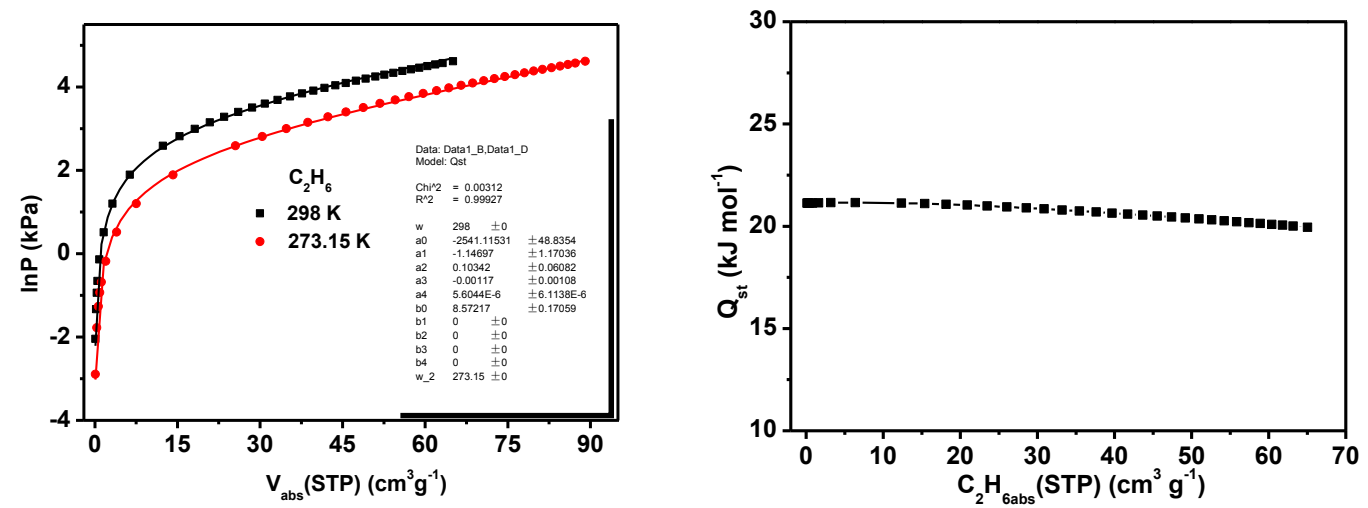

Figure S13. Fitted $\mathrm{C}_{2} \mathrm{H}_{6}$ isotherms of $1 \mathrm{a}$ measured at 273.15 and $298 \mathrm{~K}$, and the corresponding isosteric heats of adsorption $\left(Q_{\mathrm{st}}\right)$.
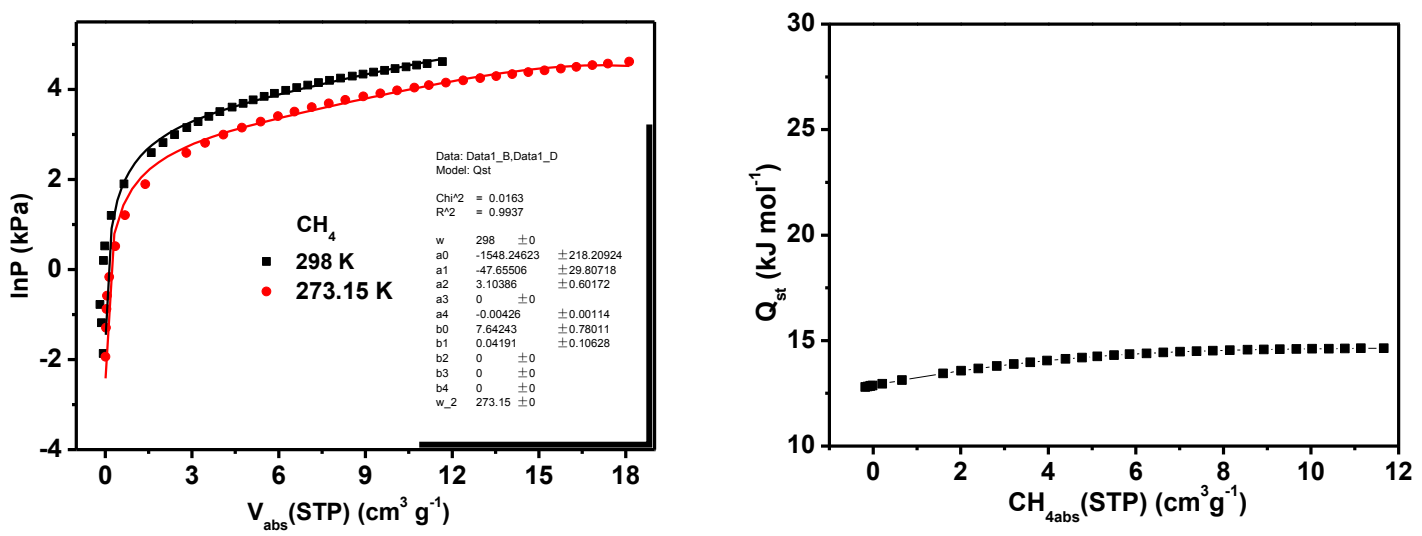

Figure S14. Fitted $\mathrm{CH}_{4}$ isotherms of $1 \mathrm{a}$ measured at 273.15 and $298 \mathrm{~K}$, and the corresponding isosteric heats of adsorption $\left(Q_{\mathrm{st}}\right)$.
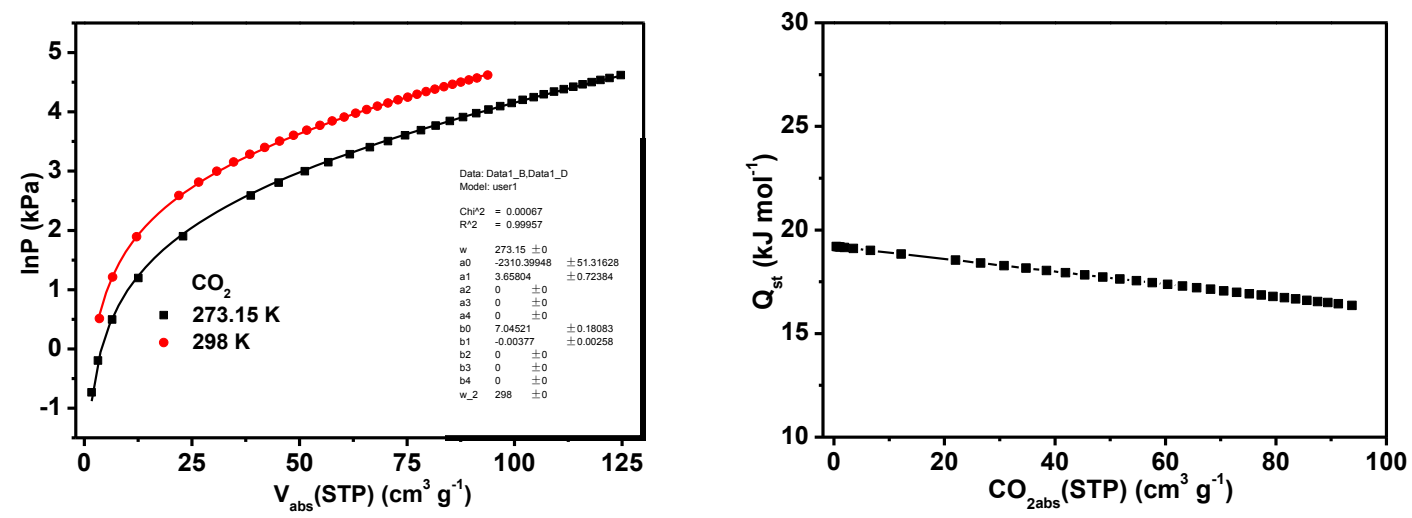

Figure S15. Fitted $\mathrm{CO}_{2}$ isotherms of $1 \mathrm{a}$ measured at 273.15 and $298 \mathrm{~K}$, and the corresponding isosteric heats of adsorption $\left(Q_{\mathrm{st}}\right)$. 

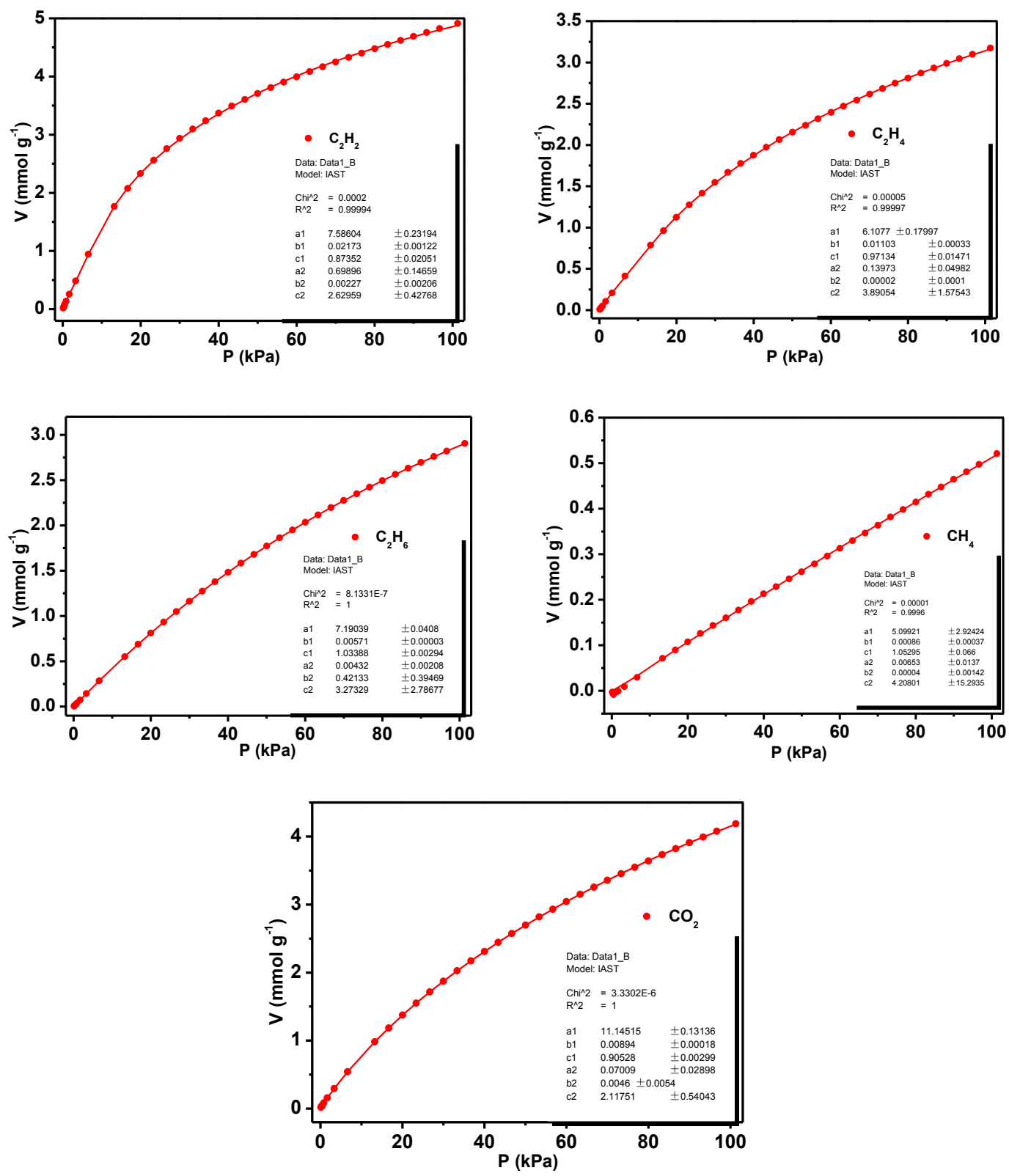

Figure S16. Fitted adsorption isotherms of 1a by dual L-F model.

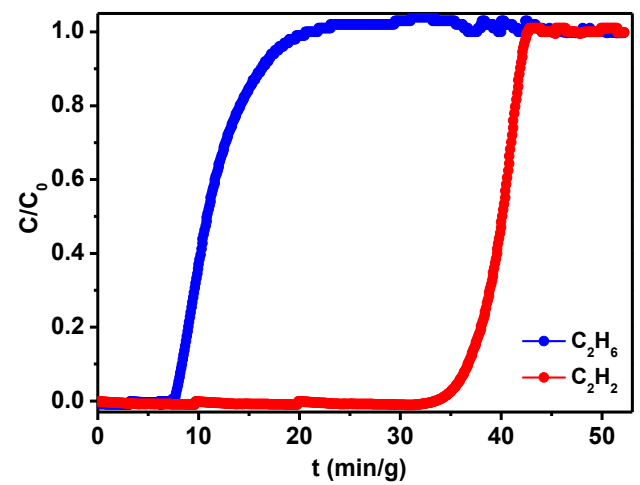

Figure S17. Breakthrough curve of 1a for equimolar $\mathrm{C}_{2} \mathrm{H}_{2}-\mathrm{C}_{2} \mathrm{H}_{6}$ mixture. 
Table S1. Crystal Data and Structure Refinements for $\mathbf{1 .}$

\begin{tabular}{ll}
\hline Chemical formula & $\mathrm{C}_{9} \mathrm{H}_{10} \mathrm{CaN}_{5} \mathrm{O}_{3}$ \\
Formula weight & 276.30 \\
$T(\mathrm{~K})$ & $150(2)$ \\
Crystal system, Space group & Trigonal, $R-3$ \\
$a(\AA)$ & $31.8746(14)$ \\
$b(\AA)$ & $31.8746(14)$ \\
$c(\AA)$ & $8.8989(4)$ \\
$\alpha\left(^{\circ}\right)$ & 90 \\
$\beta\left(^{\circ}\right)$ & 90 \\
$\gamma\left({ }^{\circ}\right)$ & 120 \\
$V\left(\AA^{3}\right)$ & $7829.9(8)$ \\
$Z$ & 18 \\
$\left.D_{\text {calcd. }}{ }^{\circ} \cdot \mathrm{g}^{-3} \mathrm{~cm}^{-3}\right]$ & 1.055 \\
$\mu\left(\mathrm{mm}{ }^{-1}\right)$ & 0.367 \\
$\mathrm{Reflns} \mathrm{collected} / \mathrm{unique} / R_{\text {int }}$ & $37297 / 3152 / 0.0545$ \\
$\mathrm{Goof}^{\mathrm{a}}$ & 1.031 \\
$R_{1}^{\mathrm{a}}, w R_{2}{ }^{\mathrm{b}}[\mathrm{I}>2 \sigma]$ & $\mathrm{R}_{1}=0.0955, \mathrm{wR}_{2}=0.2064$ \\
$R_{1}^{\mathrm{a}}, w R_{2}{ }^{\mathrm{b}}(\mathrm{all}$ data $)$ & $\mathrm{R}_{1}=0.1028, \mathrm{wR}_{2}=0.2092$ \\
\hline${ }^{a} \mathrm{R}_{1}=\Sigma\left(\left|\mathrm{F}_{\mathrm{o}}\right|-\mid \mathrm{F}_{\mathrm{c}}\right) / \Sigma\left|\mathrm{F}_{\mathrm{o}}\right|{ }^{b} \mathrm{R}_{2}=\left[\Sigma \mathrm{w}\left(\mathrm{F}_{\mathrm{o}}{ }^{2}-\mathrm{F}_{\mathrm{c}}{ }^{2}\right)^{2} / \Sigma \mathrm{w}\left(\mathrm{F}_{\mathrm{o}}{ }^{2}\right)^{2}\right]^{1 / 2}$ \\
\hline
\end{tabular}

Table S2. Selected Bond Lengths $[\AA]$ and Angles $\left[{ }^{\circ}\right]$ for $\mathbf{1}$.

\begin{tabular}{|l|l|l|l|}
\hline $\mathrm{Ca}(1)-\mathrm{O}(3)$ & $2.298(6)$ & $\mathrm{O}(1)-\mathrm{Ca}(1)-\mathrm{N}(1)$ & $73.68(13)$ \\
\hline $\mathrm{Ca}(1)-\mathrm{O}(1)$ & $2.310(4)$ & $\mathrm{O}(1) \# 1-\mathrm{Ca}(1)-\mathrm{N}(1)$ & $80.10(13)$ \\
\hline $\mathrm{Ca}(1)-\mathrm{O}(1) \# 1$ & $2.441(4)$ & $\mathrm{O}(2) \# 1-\mathrm{Ca}(1)-\mathrm{N}(1)$ & $115.29(18)$ \\
\hline $\mathrm{Ca}(1)-\mathrm{O}(2) \# 1$ & $2.458(5)$ & $\mathrm{O}(3)-\mathrm{Ca}(1)-\mathrm{N}(3) \# 2$ & $96.7(2)$ \\
\hline $\mathrm{Ca}(1)-\mathrm{N}(1)$ & $2.464(4)$ & $\mathrm{O}(1)-\mathrm{Ca}(1)-\mathrm{N}(3) \# 2$ & $78.59(14)$ \\
\hline $\mathrm{Ca}(1)-\mathrm{N}(3) \# 2$ & $2.480(4)$ & $\mathrm{O}(1) \# 1-\mathrm{Ca}(1)-\mathrm{N}(3) \# 2$ & $118.75(15)$ \\
\hline $\mathrm{Ca}(1)-\mathrm{N}(2) \# 3$ & $2.580(4)$ & $\mathrm{O}(2) \# 1-\mathrm{Ca}(1)-\mathrm{N}(3) \# 2$ & $94.27(18)$ \\
\hline $\mathrm{O}(3)-\mathrm{Ca}(1)-\mathrm{O}(1)$ & $85.94(19)$ & $\mathrm{N}(1)-\mathrm{Ca}(1)-\mathrm{N}(3) \# 2$ & $150.20(15)$ \\
\hline $\mathrm{O}(3)-\mathrm{Ca}(1)-\mathrm{O}(1) \# 1$ & $119.9(2)$ & $\mathrm{O}(3)-\mathrm{Ca}(1)-\mathrm{N}(2) \# 3$ & $161.50(19)$ \\
\hline $\mathrm{O}(1)-\mathrm{Ca}(1)-\mathrm{O}(1) \# 1$ & $143.86(14)$ & $\mathrm{O}(1)-\mathrm{Ca}(1)-\mathrm{N}(2) \# 3$ & $76.33(14)$ \\
\hline $\mathrm{O}(3)-\mathrm{Ca}(1)-\mathrm{O}(2) \# 1$ & $80.5(2)$ & $\mathrm{O}(1) \# 1-\mathrm{Ca}(1)-\mathrm{N}(2) \# 3$ & $74.21(13)$ \\
\hline $\mathrm{O}(1)-\mathrm{Ca}(1)-\mathrm{O}(2) \# 1$ & $163.88(16)$ & $\mathrm{O}(2) \# 1-\mathrm{Ca}(1)-\mathrm{N}(2) \# 3$ & $117.79(17)$ \\
\hline $\mathrm{O}(1) \# 1-\mathrm{Ca}(1)-\mathrm{O}(2) \# 1$ & $52.12(13)$ & $\mathrm{N}(1)-\mathrm{Ca}(1)-\mathrm{N}(2) \# 3$ & $78.06(15)$ \\
\hline $\mathrm{O}(3)-\mathrm{Ca}(1)-\mathrm{N}(1)$ & $92.1(2)$ & $\mathrm{N}(3) \# 2-\mathrm{Ca}(1)-\mathrm{N}(2) \# 3$ & $85.06(14)$ \\
\hline
\end{tabular}

Symmetry codes: \#1 4/3-y, x-y+2/3, z-1/3; \#2 4/3-y, x-y+2/3, z+2/3; \#3 2/3-x+y, 4/3-x, z+1/3. 
Table S3. Gas Adsorption Results.

\begin{tabular}{cccccccccccccc}
\hline $1 \mathrm{a}$ & $\begin{array}{c}\mathrm{S}_{\mathrm{BET}} \\
\left(\mathrm{m}^{2} \mathrm{~g}^{-1}\right)\end{array}$ & $\begin{array}{c}\text { Pore } \\
\text { size } \\
(\AA)\end{array}$ & \multicolumn{3}{c}{ Adsorption uptake $\left(\mathrm{cm}^{3} \mathrm{~g}^{-1}\right)$} & \multicolumn{5}{c}{$Q_{\text {st }}\left(\mathrm{kJ} \mathrm{mol}^{-1}\right)$} & & \\
& & & $\mathrm{C}_{2} \mathrm{H}_{2}$ & $\mathrm{C}_{2} \mathrm{H}_{4}$ & $\mathrm{C}_{2} \mathrm{H}_{6}$ & $\mathrm{CH}_{4}$ & $\mathrm{CO}_{2}$ & $\mathrm{C}_{2} \mathrm{H}_{2}$ & $\mathrm{C}_{2} \mathrm{H}_{4}$ & $\mathrm{C}_{2} \mathrm{H}_{6}$ & $\mathrm{CH}_{4}$ & $\mathrm{CO}_{2}$ \\
\hline $195 \mathrm{~K}$ & 553.8 & $5-10$ & & & & & 209.5 & & & & & \\
$273 \mathrm{~K}$ & & & 145.8 & 93.1 & 89.1 & 18.1 & 124.7 & & & & & \\
$298 \mathrm{~K}$ & & & 110.0 & 71.1 & 65.1 & 11.6 & 93.7 & 28.8 & 20.3 & 21.1 & 12.8 & 19.2 \\
\hline
\end{tabular}

Table S4. Comparison of $Q_{\text {st }}$, uptake and gas selectivity of $\mathrm{C}_{2} \mathrm{H}_{2}$ in MOFs at $298 \mathrm{~K}$.

\begin{tabular}{|c|c|c|c|c|c|c|c|c|}
\hline \multirow[b]{2}{*}{ MOFs } & \multirow{2}{*}{$\begin{array}{c}\mathrm{C}_{2} \mathrm{H}_{2} \\
\text { Uptake } \\
\left(\mathrm{mmol} \mathrm{g}^{-1}\right)\end{array}$} & \multirow{2}{*}{$\begin{array}{c}\mathrm{C}_{2} \mathrm{H}_{2} \\
Q_{\mathrm{st}} \\
\left(\mathrm{kJ} \mathrm{mol}^{-1}\right)\end{array}$} & \multicolumn{5}{|c|}{ Gas selectivity } & \multirow[b]{2}{*}{ Ref. } \\
\hline & & & $\begin{array}{c}\mathrm{C}_{2} \mathrm{H}_{2} / \\
\mathrm{CO}_{2}\end{array}$ & $\begin{array}{l}\mathrm{C}_{2} \mathrm{H}_{2} / \\
\mathrm{C}_{2} \mathrm{H}_{4}\end{array}$ & $\begin{array}{c}\mathrm{C}_{2} \mathrm{H}_{2} / \\
\mathrm{CH}_{4}\end{array}$ & $\begin{array}{c}\mathrm{C}_{2} \mathrm{H}_{4} / \\
\mathrm{CH}_{4}\end{array}$ & $\begin{array}{l}\mathrm{CO}_{2} / \\
\mathrm{CH}_{4}\end{array}$ & \\
\hline $1 \mathbf{a}$ & 4.91 & 28.9 & 1.7 & 2.8 & 33.3 & 12.1 & 16.5 & $\begin{array}{l}\text { This } \\
\text { work }\end{array}$ \\
\hline $\mathrm{Fe}_{2}(\mathrm{dobdc})$ & 6.8 & 47 & -- & 1.87 & 700 & 300 & -- & S1 \\
\hline NOTT-300 & $6.34^{\mathrm{a}}$ & $32^{\mathrm{a}}$ & -- & $2.30^{\mathrm{a}}$ & $1000^{\mathrm{a}}$ & $380^{\mathrm{a}}$ & -- & S2 \\
\hline ATC-Cu & 5.01 & 79.1 & 53.6 & -- & -- & -- & -- & S3 \\
\hline FJU-6-TATB & $4.91^{\mathrm{c}}$ & $29^{c}$ & -- & $3.1^{\mathrm{c}}$ & -- & -- & -- & S4 \\
\hline NCU-100a & 4.57 & 60.5 & -- & -- & -- & -- & -- & S5 \\
\hline UTSA-100a & $4.27^{\mathrm{c}}$ & $22^{\mathrm{c}}$ & -- & $10.7^{\mathrm{c}}$ & -- & -- & -- & S6 \\
\hline ZU-12-Ni & 4.21 & 40 & -- & 6.1 & 22.7 & -- & -- & S7 \\
\hline ZJU-74a & $3.83^{\mathrm{c}}$ & $45^{c}$ & $36.5^{\mathrm{c}}$ & $24.1^{\mathrm{c}}$ & -- & -- & -- & S8 \\
\hline NUC-2 & 2.99 & 39.8 & -- & 8.1 & -- & -- & -- & S9 \\
\hline $\mathrm{NbU}-1$ & 2.72 & 38.3 & -- & 5.9 & -- & -- & -- & S10 \\
\hline JLU-MOF66 & 2.45 & 35.6 & -- & -- & 86.2 & -- & 9.4 & S11 \\
\hline PCP-NH2-ipa & 1.93 & 26.8 & -- & -- & -- & -- & -- & S12 \\
\hline M'MOF-3a & $1.9^{b}$ & $27.1^{\mathrm{b}}$ & -- & $1.9^{\mathrm{b}}$ & -- & -- & -- & S13 \\
\hline Cd-NP & 0.43 & -- & -- & -- & -- & -- & -- & S14 \\
\hline MOF-5 & $1.16^{\mathrm{b}}$ & $16.5^{\mathrm{b}}$ & -- & -- & -- & -- & -- & S15 \\
\hline ZIF-8 & $1.14^{\mathrm{b}}$ & $13.3^{\mathrm{b}}$ & -- & -- & -- & -- & -- & S15 \\
\hline
\end{tabular}




\begin{tabular}{|c|c|c|c|c|c|c|c|c|}
\hline NOTT-101a & 8.21 & -- & 9 & -- & 36.9 & 30 & -- & $\mathrm{S} 16$ \\
\hline $\mathrm{Cu}_{0.5}(\mathrm{tztp})_{0.5}$ & 5.0 & 38.3 & 2.7 & -- & 23 & 11.3 & 6.5 & $\mathrm{~S} 17$ \\
\hline SNNU-17 & 1.69 & 30.5 & 1.2 & -- & 20.3 & 9.4 & 12 & $\mathrm{~S} 18$ \\
\hline Ni(dpip) & 3.73 & 41.7 & 2 & -- & 18.5 & 7.5 & 9.1 & $\mathrm{~S} 19$ \\
\hline$\left[\mathrm{In}_{2}(\mathrm{~L})(\mathrm{OH})_{2}\right]$ & 3.79 & 32 & -- & -- & 22 & 13 & 5 & $\mathrm{~S} 20$ \\
\hline ZJNU-63 & 1.76 & 32.9 & -- & -- & 13.1 & 7.0 & 3.5 & $\mathrm{~S} 21$ \\
\hline Sc-EBTC & 3.94 & 22.6 & -- & -- & 12.5 & 11.3 & 3.8 & $\mathrm{~S} 22$ \\
\hline SNNU-5-In & 3.42 & 28.9 & -- & -- & 10 & 7.4 & 3.9 & $\mathrm{~S} 23$ \\
\hline NbU-10 & $2.79^{\mathrm{a}}$ & $31.3^{\mathrm{a}}$ & $2.8^{\mathrm{a}}$ & $6.4^{\mathrm{a}}$ & -- & -- & -- & $\mathrm{S} 24$ \\
\hline BSF-1 & 2.35 & 31 & 3.3 & 2.3 & -- & -- & -- & $\mathrm{S} 25$ \\
\hline MUF-17 & $3.01^{\mathrm{a}}$ & $49.5^{\mathrm{a}}$ & $6^{\mathrm{a}}$ & $8.73^{\mathrm{a}}$ & -- & -- & -- & $\mathrm{S} 26$ \\
\hline M'MOF-2a & -- & 37.7 & 1.89 & 1.93 & -- & -- & -- & $\mathrm{S} 13$ \\
\hline ZJU-199a & $5.71^{\mathrm{c}}$ & $38.5^{\mathrm{c}}$ & $4.0^{\mathrm{c}}$ & -- & $27.3^{\mathrm{c}}$ & -- & -- & $\mathrm{S} 27$ \\
\hline SNNU-27-Fe & 8.14 & 24.1 & 2 & -- & -- & -- & -- & $\mathrm{S} 28$ \\
\hline SNNU-26-Mn & 4.99 & 30.3 & 1.4 & -- & -- & -- & -- & $\mathrm{S} 28$ \\
\hline FJU-36a & $2.33^{\mathrm{c}}$ & $32.9^{\mathrm{c}}$ & $2.8^{\mathrm{c}}$ & -- & $17.7^{\mathrm{c}}$ & -- & -- & $\mathrm{S} 29$ \\
\hline FJU-59a & 3.03 & 35.6 & 4.3 & -- & -- & -- & -- & $\mathrm{S} 30$ \\
\hline FJU-90a & 8.03 & 25.1 & 4.3 & -- & -- & -- & -- & $\mathrm{S} 31$ \\
\hline TCuCl & 2.2 & 41 & 5.3 & -- & -- & -- & -- & $\mathrm{S} 32$ \\
\hline
\end{tabular}

*a: measured at $293 \mathrm{~K}$; b: measured at $295 \mathrm{~K}$; c: measured at $296 \mathrm{~K}$.

\section{References}

S1. Bloch, E. D.; Queen, W. L.; Krishna, R.; Zadrozny, J. M.; Brown, C. M.; Long, J. R. Hydrocarbon Separations in a Metal-Organic Framework with Open Iron(II) Coordination Sites. Science 2012, 335, 1606-1610.

S2. Yang, S.; Ramirez-Cuesta, A. J.; Newby, R.; Garcia-Sakai, V.; Manuel, P.; Callear, S. K.; Campbell, S. I.; Tang, C. C.; Schröder, M. Supramolecular binding and separation of hydrocarbons within a functionalized porous metal-organic framework, Nat. Chem. 2015, 7, 121-129. 
S3. Niu, Z.; Cui, X.; Pham, T.; Verma, G.; Lan, P. C.; Shan, C.; Xing, H.; Forrest, K. A.; Suepaul, S.; Space, B.; Nafady, A.; Al-Enizi, A. M.; Ma, S. A MOF-based Ultra-Strong Acetylene Nano-trap for Highly Efficient $\mathrm{C}_{2} \mathrm{H}_{2} / \mathrm{CO}_{2}$ Separation, Angew. Chem. Int. Ed. 2021, 60, 5283-5288.

S4. Liu, L.; Yao, Z.; Ye, Y.; Yang, Y.; Lin, Q.; Zhang, Z.; O’Keeffe, M.; Xiang, S. Integrating the Pillared-Layer Strategy and Pore-Space Partition Method to Construct Multicomponent MOFs for $\mathrm{C}_{2} \mathrm{H}_{2} / \mathrm{CO}_{2}$ Separation, J. Am. Chem. Soc. 2020, 142, 9258-9266.

S5. Wang, J.; Zhang, Y.; Zhang, P.; Hu, J.; Lin, R.-B.; Deng, Q.; Zeng, Z.; Xing, H.; Deng, S.; Chen, B. Optimizing Pore Space for Flexible-Robust Metal-Organic Framework to Boost Trace Acetylene Removal, J. Am. Chem. Soc. 2020, 142, 9744-9751.

S6. Hu, T.-L.; Wang, H.; Li, B.; Krishna, R.; Wu, H.; Zhou, W.; Zhao, Y.; Han, Y.; Wang, X.; Zhu, W.; Yao, Z.; Xiang, S.; Chen, B. Microporous metal-organic framework with dual functionalities for highly efficient removal of acetylene from ethylene/acetylene mixtures, Nat. Commun. 2016, 6, 7328.

S7. Jiang, M.; Cui, X.; Yang, L.; Yang, Q.; Zhang, Z.; Yang, Y.; Xing, H.; A thermostable anion-pillared metal-organic framework for $\mathrm{C}_{2} \mathrm{H}_{2} / \mathrm{C}_{2} \mathrm{H}_{4}$ and $\mathrm{C}_{2} \mathrm{H}_{2} / \mathrm{CO}_{2}$ separations, Chem. Eng. J. 2018, 352, 803-810.

S8. Pei, J.; Shao, K.; Wang, J.-X.; Wen, H.-M.; Yang, Y.; Cui, Y.; Krishna, R.; Li, B.; Qian, G. A Chemically Stable Hofmann-Type Metal-Organic Framework with Sandwich-Like Binding Sites for Benchmark Acetylene Capture, Adv. Mater. 2020, 32, 1908275.

S9. Chen, H.; Zhuang, G.-L.; Fan, L.; X. Zhang.; Gao, L.-N.; Sun, D. A highly robust heterometallic $\mathrm{Tb}^{\mathrm{III}} / \mathrm{Ni}^{\mathrm{II}}$-organic framework for $\mathrm{C} 2$ hydrocarbon separation and capture, Chem. Commun. 2020, 56, 2047-2050.

S10. Li, J.; Jiang, L.; Chen, S.; Kirchon, A.; Li, B.; Li, Y.; Zhou, H.-C.; Metal-Organic Framework Containing Planar Metal-Binding Sites: Efficiently and Cost-Effectively Enhancing the Kinetic Separation of $\mathrm{C}_{2} \mathrm{H}_{2} / \mathrm{C}_{2} \mathrm{H}_{4}, J$. Am. Chem. Soc. 2019, 141, 3807-3811.

S11. Kan, L.; Li, G.; Liu, Y. Highly Selective Separation of $\mathrm{C}_{3} \mathrm{H}_{8}$ and $\mathrm{C}_{2} \mathrm{H}_{2}$ from $\mathrm{CH}_{4}$ within Two Water-Stable $\mathrm{Zn}_{5}$ Cluster-Based Metal-Organic Frameworks, ACS Appl. 
Mater. Interfaces 2020, 12, 18642-18649.

S12. Gu, Y.; Zheng, J.-J.; Otake, K.; Shivanna, M.; Sakaki, S.; Yoshino, H.; Ohba, M.;

Kawaguchi, S.; Wang, Y.; Li, F.; Kitagawa, S. Host-Guest Interaction Modulation in Porous Coordination Polymers for Inverse Selective $\mathrm{CO}_{2} / \mathrm{C}_{2} \mathrm{H}_{2}$ Separation, Angew. Chem. Int. Ed. 2021, 60, 11688-11694.

S13. Xiang, S.-C.; Zhang, Z.; Zhao, C.-G.; Hong, K.; Zhao, X.; Ding, D.-R.; Xie, M.-H.; Wu, C.-D.; M. Das, C.; Gill, R.; Thomas, K. M.; Chen, B. Rationally tuned micropores within enantiopure metal-organic frameworks for highly selective separation of acetylene and ethylene, Nat. Commun. 2011, 2, 204.

S14. Xie, Y.; Cui, H.; Wu, H.; Lin, R.-B.; Zhou, W.; Chen, B. Electrostatically Driven Selective Adsorption of Carbon Dioxide over Acetylene in an Ultramicroporous Material, Angew. Chem. Int. Ed. 2021, 60, 9604-9609

S15. Xiang, S.; Zhou, W.; Gallegos, J. M.; Liu, Y.; Chen, B. Exceptionally High Acetylene Uptake in a Microporous Metal-Organic Framework with Open Metal Sites, $J$. Am. Chem. Soc. 2009, 131, 12415-12419.

S16. Wen, H.-M.; Wang, H.; Li, B.; Cui, Y.; Wang, H.; Qian, G.; Chen, B. A Microporous Metal-Organic Framework with Lewis Basic Nitrogen Sites for High $\mathrm{C}_{2} \mathrm{H}_{2}$ Storage and Significantly Enhanced $\mathrm{C}_{2} \mathrm{H}_{2} / \mathrm{CO}_{2}$ Separation at Ambient Conditions, Inorg. Chem. 2016, $55,7214-7218$.

S17. Li, X.-Y.; Li, Y.-Z.; Ma, L.-N.; Hou, L.; He, C.-Z.; Wang, Y.-Y.; Zhu, Z. Efficient gas and alcohol uptake and separation driven by two types of channels in a porous MOF: an experimental and theoretical investigation. J. Mater. Chem. A 2020, 8, 5227-5233.

S18. Li, H.-P.; Xue, Y.-Y.; Wang, Y.; Sun, H.; Hu, M.-C.; Li, S.-N.; Jiang, Y.; Zhai, Q.-G. Regulation on Topological Architectures and Gas Adsorption for Cadmium-Azolate-Carboxylate Frameworks by the Ligand Flexibility. Cryst. Growth Des. 2021, 21, 1718-1726.

S19. $\quad$ Li, Y.-Z.; Wang, G.-D.; Ma, L.-N.; Hou, L.; Wang, Y.-Y.; Zhu, Z. Multiple Functions of Gas Separation and Vapor Adsorption in a New MOF with Open Tubular Channels. ACS Appl. Mater. Interfaces 2021, 13, 4102-4109.

S20. Zhang, B.; Guo, P.-Y.; Ma, L.-N.; Liu, B.; Hou, L.; Wang, Y.-Y.; Two Robust S15 
In(III)-Based Metal-Organic Frameworks with Higher Gas Separation, Efficient Carbon Dioxide Conversion, and Rapid Detection of Antibiotics, Inorg. Chem. 2020, 59, $5231-5239$.

S21. Bai, D.; Wang, Y.; He, M.; Gao, X.; He, Y. Structural diversities and gas adsorption properties of a family of rod-packing lanthanide-organic frameworks based on cyclotriphosphazene-functionalized hexacarboxylate derivatives, Inorg. Chem. Front., 2018, 5, 2227-2237.

S22. Zhang, J.-W.; Qu, P.; Hu, M.-C.; Li, S.-N.; Jiang, Y.-C.; Zhai, Q.-G. Topology-Guided Design for Sc-soc-MOFs and Their Enhanced Storage and Separation for $\mathrm{CO}_{2}$ and $\mathrm{C}_{2}-$ Hydrocarbons, Inorg. Chem. 2019, 58, 16792-16799.

S23. Zhang, J.-W.; Ji, W.-J.; Hu, M.-C.; Li, S.-N.; Jiang, Y.-C.; Zhang, X.-M.; P. Qu.; Zhai, Q.-G. A superstable 3p-block metal-organic framework platform towards prominent $\mathrm{CO}_{2}$ and $\mathrm{C} 1 / \mathrm{C} 2$-hydrocarbon uptake and separation performance and strong Lewis acid catalysis for $\mathrm{CO}_{2}$ fixation, Inorg. Chem. Front. 2019, 6, 813-819.

S24. Zhao, J.; Li, Q.; Zhu, X.; Li, J.; Wu, D. Highly Robust Tetranuclear Cobalt-Based 3D Framework for Efficient $\mathrm{C}_{2} \mathrm{H}_{2} / \mathrm{CO}_{2}$ and $\mathrm{C}_{2} \mathrm{H}_{2} / \mathrm{C}_{2} \mathrm{H}_{4}$ Separations, Inorg. Chem. 2020, $59,14424-14431$.

S25. Zhang, Y.; Yang, L.; Wang, L.; Duttwyler, S.; Xing, H. A Microporous Metal-Organic Framework Supramolecularly Assembled from a $\mathrm{Cu}^{\mathrm{II}}$ Dodecaborate Cluster Complex for Selective Gas Separation, Angew. Chem. Int. Ed. 2019, 58, 8145-8150.

S26. Qazvini, O. T.; Babarao, R.; Telfer, S. G. Multipurpose Metal-Organic Framework for the Adsorption of Acetylene: Ethylene Purification and Carbon Dioxide Removal, Chem. Mater. 2019, 31, 4919-4926.

S27. Zhang, L.; Zou, C.; Zhao, M.; Jiang, K.; Lin, R.; He, Y.; Wu, C.-D.; Cui, Y.; Chen, B.; Qian, G. Doubly Interpenetrated Metal-Organic Framework for Highly Selective $\mathrm{C}_{2} \mathrm{H}_{2} / \mathrm{CH}_{4}$ and $\mathrm{C}_{2} \mathrm{H}_{2} / \mathrm{CO}_{2}$ Separation at Room Temperature, Cryst. Growth Des. 2016, 16, 7194-7197.

S28. Xue, Y.-Y.; Bai, X.-Y.; Zhang, J.; Wang, Y.; Li, S.-N.; Jiang, Y.-C.; Hu, M.-C.; Zhai, Q.-G. Precise Pore Space Partitions Combined with High-Density Hydrogen-Bonding 
Acceptors within Metal-Organic Frameworks for Highly Efficient Acetylene Storage and Separation. Angew. Chem. Int. Ed. 2021, 60, 10122-10128.

S29. Liu, L.; Yao, Z.; Ye, Y.; Chen, L.; Lin, Q.; Yang, Y.; Zhang, Z.; Xiang, S. Robustness, Selective Gas Separation, and Nitrobenzene Sensing on Two Isomers of Cadmium Metal-Organic Frameworks Containing Various Metal-O-Metal Chains, Inorg. Chem. 2018, 57, 12961-12968.

S30. Guo, J.; Li, Z.; Liu, L.; Ye, Y.; Chen, T.; Zhang, M.; Ke, Z.; Xiang, S.; Lv, Y.; Yao, Z. A metal-organic framework with double interpenetrated frameworks for effective $\mathrm{C}_{2} \mathrm{H}_{2} / \mathrm{CO}_{2}$ separation, Inorg. Chem. Commun. 2020, 112, 107721.

S31. Ye, Y.; Ma, Z.; Lin, R.-B.; Krishna, R.; Zhou, W.; Lin, Q.; Zhang, Z.; Xiang, S.; Chen, B. Pore Space Partition within a Metal-Organic Framework for Highly Efficient $\mathrm{C}_{2} \mathrm{H}_{2} / \mathrm{CO}_{2}$ Separation, J. Am. Chem. Soc. 2019, 141, 4130-4136.

S32. Mukherjee, S.; He, Y.; Franz, D.; Wang, S.-Q.; Xian, W.-R.; Bezrukov,A. A.; Space, B.; Xu, Z.; He, J.; Zaworotko, J, M. Halogen- $\mathrm{C}_{2} \mathrm{H}_{2}$ Binding in Ultramicroporous Metal-Organic Frameworks (MOFs) for Benchmark $\mathrm{C}_{2} \mathrm{H}_{2} / \mathrm{CO}_{2}$ Separation Selectivity, Chem. Eur. J. 2020, 26, 4923-4929. 